\title{
“Crystal Collimator" Measurement of CESR particle-beam Source Size
}

\author{
K. D. Finkelstein, Ivan Bazarov, Jeffrey White, Peter Revesz \\ Cornell High Energy Synchrotron Source (CHESS), Wilson Laboratory, \\ Cornell University, Ithaca, New York 14853
}

\begin{abstract}
We have measured electron and positron beam source size at CHESS when the Cornell Electron Storage Ring (CESR) is run dedicated for the production of synchrotron radiation. Horizontal source size at several beamlines is expected to shrink by a factor of two but synchrotron (visible) light measurements only provide the vertical size. Therefore a "crystal collimator" using two Bragg reflection in dispersive $(+,+)$ orientation has been built to image the horizontal (vertical) source by passing x-rays parallel to within 5 microradians to an imaging screen and camera. With the "crystal collimator" we observe rms sizes of $1.2 \mathrm{~mm}$ horizontal by $0.28 \mathrm{~mm}$ vertical, in good agreement with the $1.27 \mathrm{~mm}$ size calculated from lattice functions, and $0.26 \mathrm{~mm}$ observed using a synchrotron light interferometer.
\end{abstract}

\section{INTRODUCTION}

The Cornell Electron Storage Ring (CESR) began periodic, dedicated operation to produce synchrotron radiation for CHESS in January 2003. The storage ring, usually operated as an e+/e- collider, is optimized for high luminosity, which favors large horizontal emittance and maximum beam currents. For CHESS operation, the twobeams lattice was adjusted to: avoid electron-positron collisions (increasing beam lifetime to $>12$ hours), minimize dispersion near CHESS source points (reducing horizontal emittance by $\sim 2$ ), and limit currents to $200 \mathrm{~mA} / \mathrm{beam}$.

The goals of work reported here are to: measure particle beam horizontal source size, develop a simple real-time monitor for transverse source dimensions, and corroborate synchrotron light interferometer (IF) measurements [1] of vertical source size. Before this study, only estimates of the vertical size at CHESS source points $\left(\mathrm{S}_{\mathrm{i}}\right)$ were available by scaling IF measurements by $\sqrt{ }\left[\beta_{\mathrm{V}}\left(\mathrm{S}_{\mathrm{i}}\right) / \beta_{\mathrm{V}}(\mathrm{IF})\right]$, where $\beta_{\mathrm{V}}$ is the measured vertical beta function at two locations. On the other hand, because horizontal source dimensions are 5 to 10 times larger than vertical, visible light measurements have not available with the desired precision.

To our knowledge the method underlying the x-ray monitor described below has been employed for scanning type measurements twice in the past. First, at the Daresbury SRS by M. Hart and D.P. Siddons[2], and again more recently using an undulator source at SPRING8 by Y. Kohmura and co-workers[3]. In contrast to previous work, we demonstrate an imaging system designed as a continuous source size monitor that is simple enough to operate for long periods with little human intervention.

\section{EXPERIMENTAL METHOD}

An image of the horizontal (vertical) source is made using successive Bragg reflections set in dispersive (antiparallel, or ++ ) orientation. Results were obtained using filtered bendmagnet radiation to limit heating of the first crystal. This method should work as well with monochromatic radiation and in reference [3] the dispersive reflection follows one or more double-bounce monochromators.

Two experimental arrangements were tested during successive CHESS run periods. The first uses the crystal illustrated at left in Figure. 1 with reflection $(4,-2,-2)$ following $(2,2,-4)$. The second configuration is shown at right in Figure. 1. It uses standard monochromator crystals, and the complete imaging system: crystals, piezo driven 
adjustment of the second crystal, fluorescent screen and camera are mounted to fit on the phi spindle of a Huber 4circle diffractometer.

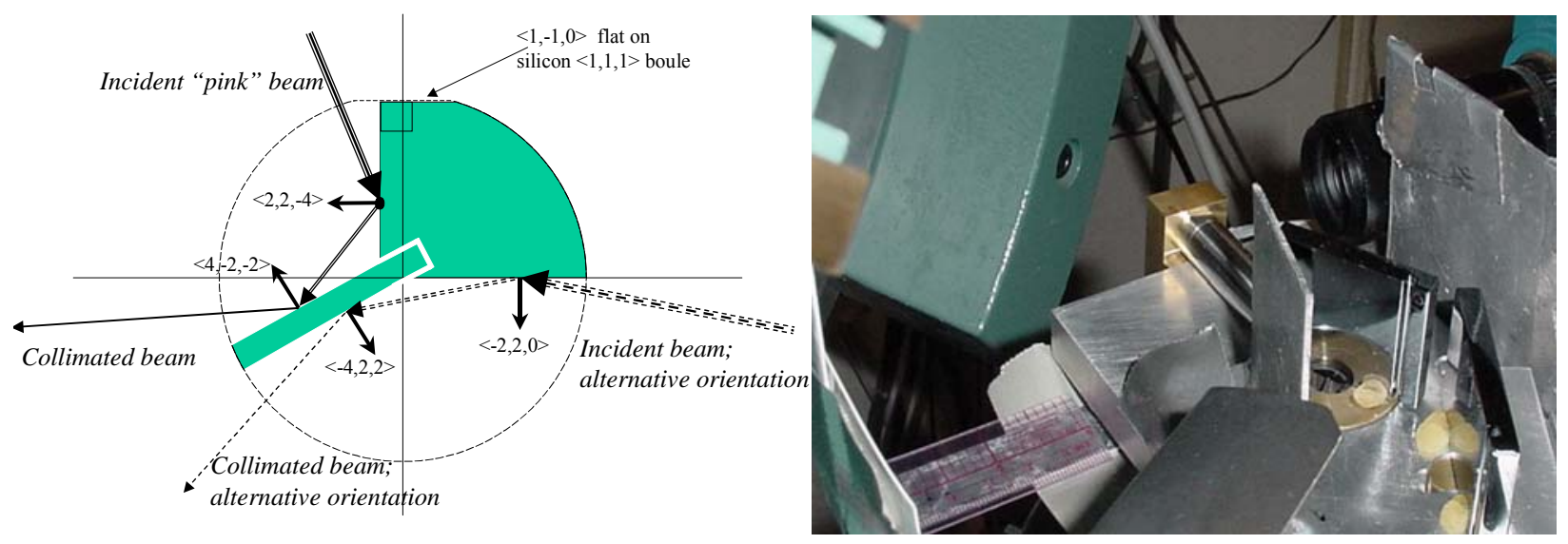

FIGURE 1. Crystal collimators used at CHESS. The device illustrated at left was used in the measurements reported here. The shaded area shows crystal cut from a $20 \mathrm{~mm}$ thick disk of float zone silicon with $<1,1,1>$ direction pointing out of the figure. The configuration permits a choice of two sets of dispersive reflections. The order of reflection is not important. The photo at right shows a very compact device recently tested. It uses standard CHESS monochromator crystals and mounts on the PHI axis of a large HUBER 4-circle diffractometer. For either device, the incident beam is filtered to reduce power on the first crystal. With bendmagnet radiation, heating effects were not apparent, when CHESS operated at $200 \mathrm{~mA}$ and $5.3 \mathrm{GeV}$, after the beam passed through 750 microns of beamline beryllium windows and 750 microns of highly oriented pyrolytic graphite.

The principle of the method used to determine horizontal source size is illustrated in Figure 2. where the Dumond diagram[4] explains the collimation effect. In the anti-parallel crystal arrangement x-rays diffract in a very narrow wavelength band over an angular range determined by the overlap of Darwin widths for the two reflections. For broad bandwidth radiation, the angular acceptance is roughly the larger of the two Darwin widths. Numbers associated with three sets of reflections chosen from in these experiments are given in Table 1. It may be worth pointing out that because of refraction at the air-crystal interface dispersive reflection often passes little or no harmonics, and, in the case of symmetric reflections diffraction occurs when the sum of Bragg angles is slightly less than the angle between the normal to each crystal surface.

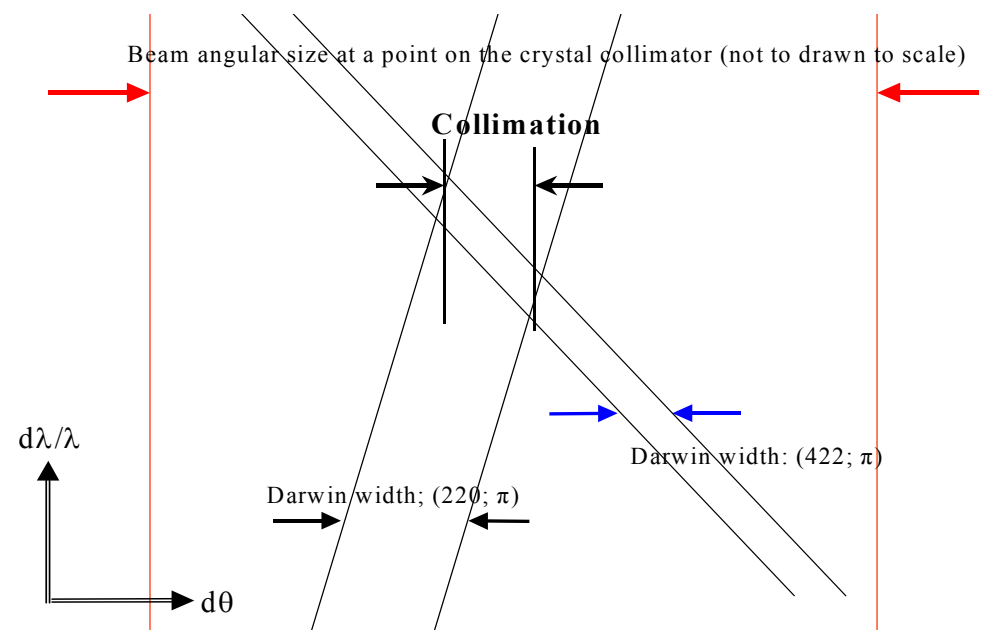

Figure 2. Dumond diagram representation for crystal collimator used to measure horizontal source size. The acceptance of two Bragg reflections, at a common wavelength in dispersive orientation, creates an overlapping region of very small angular size. The outer vertical lines (not drawn to scale) indicate the angular spread of the incident beam at a point on the collimator crystal, from a single electron traveling through the CESR bendmagnet at $5.3 \mathrm{GeV}$; about 190 micro radians. 
The collimation of reflecting crystal pairs illustrated in Figure. 1 is tabulated in Table. 1. These double reflections provide very high angular selectivity. The polarization designation $\sigma,(\pi)$ apply to measurement of vertical (horizontal) source size. In practice, an aluminum triangle is used to set the angle between crystal surfaces to 180 degrees minus the sum of Bragg angles. The device is then rotated about an axis coincident with the first surface until the wavelength selected satisfies Bragg's law for both crystals.

Table 1. Properties of Crystal Collimator Reflections.

\begin{tabular}{|c|c|c|c|c|c|c|c|c|}
\hline \multirow[t]{2}{*}{ Reflection pair } & \multirow[t]{2}{*}{$\begin{array}{c}\text { Energy } \\
(\mathrm{KeV})\end{array}$} & \multirow[t]{2}{*}{$\begin{array}{l}\text { Bragg angle } \\
\text { (degrees) }\end{array}$} & \multicolumn{2}{|c|}{$\begin{array}{l}\text { Darwin width } \\
\text { (microradians) }\end{array}$} & \multicolumn{2}{|c|}{ Peak Reflectivity } & \multicolumn{2}{|c|}{$\begin{array}{l}\text { Effective collimation }+ \\
\text { (FWHM-microradians) }\end{array}$} \\
\hline & & & $\sigma$ & $\pi$ & $\sigma$ & $\pi$ & $\sigma$ & $\pi$ \\
\hline$(2,2,-4 ; 4,-2,-2)$ & 11.185 & 30 & 8.4 & 4.2 & 0.977 & 0.905 & 4.2 & 2.1 \\
\hline$\{-2,2,0 \&$ & 17.086 & 10.895 & 11.6 & 10.8 & 0.995 & 0.992 & & \\
\hline $4,2,2\}$ & 46 & 19.105 & & & & & 6.2 & 5.8 \\
\hline$(4,4,0 ; 4,4,0)$ & 12.915 & 30 & 6.24 & 3.17 & 0.987 & 0.93 & 3.2 & 1.6 \\
\hline
\end{tabular}

$\uparrow$ Estimate ignores very small increase in measured source size due to extinction effect in diffraction.

\section{SOURCE SIZE MEASUREMENTS AT BEND MAGNET STATION B2}

The crystal collimator in Figure 1 (left) was mounted on the phi circle of a HUBER 511 Eulerian Cradle with phi axis along (111) and located where the incident beam hits the $(2,2,-4)$ face. The crystal is adjusted for reflection from $(2,2,-4)$ planes at a Bragg angle approximately 30 degrees. The $(4,-2,-2)$ angle is then adjusted, using a piezoelectric actuator, until the double reflection is intercepted by a fluorescent screen that is shielded from direct scatter off the first crystal. The x-ray intensity on the fluorescent screen was low, but the possibility of artificial image enlargement (bloom) was checked by observing the image as white beam slits limiting the beam normal to the measurement direct are closed. The screen is viewed by an Astrovid Stellacam video camera [5] through a mirror. To switch between horizontal and vertical source measurement the cradle chi axis is rotated $90^{\circ}$ about the incident beam, changing the collimator scattering plane from horizontal to vertical.

Data obtained when the "Crystal Collimator" is used to image the horizontal (Fig. 3a), and vertical (Fig. 3b) size of the CHESS B line bend magnet source approximately 15 meters upstream. In the horizontal source size measurement a semi-continuous, linearly increasing background signal was subtracted to produce a symmetric peak from the relatively weak diffraction image.
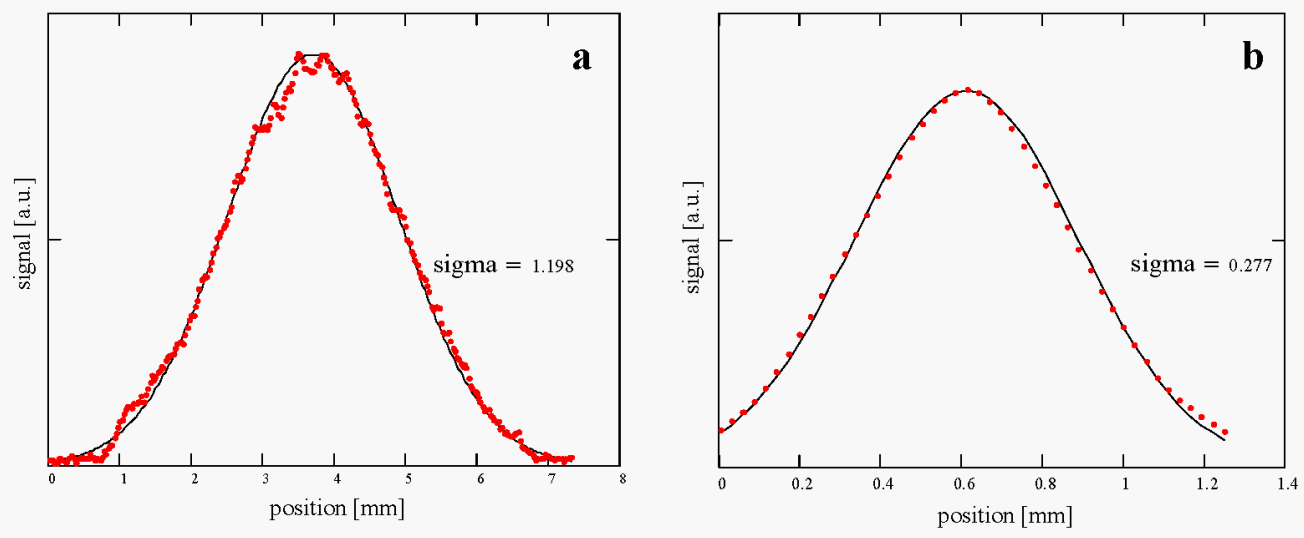

Figure 3. Measured beam profiles obtained by using crystal collimator on left side in Figure. 1. Measure values for horizontal (a) and vertical (b) source size at B2 station are obtained by a fit (solid line) to the peaks shown. Fine structure in (a) is due to defects on the fluorescent screen. As explained below, the results are in close agreement with estimates based on other methods. 


\section{COMPARISON BETWEEN MEASUREMENT AND CALCULATION}

Results of cesrv lattice code calculations for the lattice used during the first dedicated CHESS run are summarized in Table 2. Twiss parameters at the measurement location are given along with calculated values of horizontal emittance for typical CHESS parasitic operation during high energy physics.

Table 2. Calculated lattice parameters for dedicated and parasitic operation

\begin{tabular}{|l|l|l|l|l|}
\hline Parameter & CHESS dedicated run & & CHESS parasitic operation & \\
\hline Lattice designation & CD ZSQ1_15T_3s 3 & & $3 \mathrm{~s}$ v2 & \\
\hline Horiz emittance(nm-rad) & $120(\mathrm{e}-)$ & $93(\mathrm{e}+)$ & $225(\mathrm{e}-)$ & $250(\mathrm{e}+)$ \\
\hline Energy spread & 0.00063 & 0.00069 & 0.0007 & 0.0007 \\
\hline B line Twiss parameters: & & & & \\
\hline Beta (meters) & 10.77 (horizontal) & $10.35($ vert.) & 25.33 (horizontal) & 11.91 (vert.) \\
\hline Eta (meters) & 0.89 " & 0.007 “ & 1.99 “ & N/a \\
\hline Source size (rms; mm) & 1.27 " & & 2.65 “ & \\
\hline
\end{tabular}

The "crystal collimator" measure of source vertical width, $0.28 \mathrm{~mm}(\mathrm{rms})$, is in good agreement with $0.26 \mathrm{~mm}$, obtained by optical interferometer measurements scaled by the square root of the ratio of beta-functions at the two locations.

Vertical emittance is not calculated by cesrv since it is primarily determined by the amount of coupling between horizontal and vertical particle motions. The measured values of vertical source size suggest a larger than expected vertical emittance of $7 \mathrm{~nm}$-rad. The cause of this anomaly has not as yet been identified and is a subject of future machine studies. Once this problem is resolved, the dedicated CHESS lattice should provide smaller horizontal size as well as vertical emittance up to the point when elastic collisions in the bunch (Touschek effect) become the lifetime limiting factor.

\section{ACKNOWLEDGMENTS}

We thank the CHESS operations staff for support with this work, and Stuart Peck and Don Hartill of the Cornell Laboratory of Experimental Particle Physics. This work is based upon research conducted at the Cornell High Energy Synchrotron Source (CHESS) which is supported by the National Science Foundation and the National Institutes of Health/National Institute of General Medical Sciences under award DMR 9713424.

\section{REFERENCES}

1. Matsuhashi, T., "Beam Profile and Size Measurement by SR Interferometers", in Beam Measurement, Proc.Joint USCERN-Japan-Russia School on Particle Accelerators, Montreux, Switzerland, 11-20 May 1998, World Scientific, Singapore pp. 399-427

2. Hart. M., and Siddons, D.P., Nuclear Instruments and Methods 204, 219-221 (1982).

3. Kohumura, Y., Suzuki, Y., Awaji, M., Tanaka, T., Hara, T., Goto, S., Ishikawa, T., Nuclear Instruments and Methods in Physics Research A 452, 343-350 (2000).

4. Dumond, J.W.M., Physical Review 52, 872-883 (1937).

5. Astrovid Stellacam is a trademark of Adirondack Video Astronomy. This CCD camera uses a 50mm Tamron lens and $25 \mathrm{~mm}$ extender to produce an image with resolution 19.8 microns/CCD pixel. The lens to screen distance is approximately $140 \mathrm{~mm}$. 